\title{
Multiple spatially specific enhancers are required to reconstruct the pattern of Hox-2.6 gene expression
}

\author{
Jenny Whiting, Heather Marshall, Martyn Cook, Robb Krumlauf, ${ }^{1}$ Peter W.J. Rigby, David Stott, ${ }^{2}$ \\ and Rudolf K. Allemann \\ Laboratory of Eukaryotic Molecular Genetics, Medical Research Council National Institute for Medical Research, \\ London, NW7 1AA England
}

\begin{abstract}
Murine Hox genes are organized into four clusters that share many features with the homeotic clusters of Drosophila. This evolutionary conservation and the clear relationships between the position of a gene within a cluster and its expression pattern have led to the suggestion that the structure of the cluster is essential for proper regulation. Using a Hox-2.6-lacZ reporter gene in transgenic mice we have shown that the overall expression pattern of the endogenous $H o x-2.6$ gene can be reconstructed when it is isolated from the complex. The transgene was expressed in the proper tissues, with the correct spatial distribution and temporal pattern. Furthermore, direct comparison by in situ hybridization revealed that the levels of transgene expression are similar to those of the endogenous gene. This has allowed us to define three elements that regulate particular aspects of the Hox-2.6 pattern, two of which act as spatially specific enhancers. One enhancer, region $A$, directed expression only in the neural tube, whereas the other, region $\mathrm{C}$, specified the majority of the Hox-2.6 pattern. Both were also capable of imposing the correct boundaries of expression on heterologous promoters. The definition of such elements will allow the characterization of the trans-acting factors that mediate spatial regulation in the mammalian embryo.
\end{abstract}

[Key Words: Homeo box genes; transgenic mice; transcriptional regulation; pattern formation; spatially specific enhancers; Hox-2.6]

Received July 18, 1991; revised version accepted August 26, 1991.

The establishment of the body plan of the mammalian embryo requires regulatory genes to be expressed in precisely controlled spatial locations so that their products can function appropriately in the patterning process. Given the absence of appropriate genetic screens in mammals, the elucidation of the mechanisms involved in this regulatory cascade initially depends on the definition of the cis-acting sequences that mediate spatial regulation. These elements could then be used to identify the cognate trans-acting factors, the activity of which must be regulated in response to signals generated at the previous level of the hierarchy that controls pattern formation. Homeo box genes are thought to be involved in this process and themselves encode transcription factors, thereby providing an ideal system to study such developmental problems.

The vertebrate Hox family is a group of $35-40$ genes, organized in four chromosomal clusters (for review, see Kessel and Gruss 1990), which contain a homeo box mo-

\footnotetext{
${ }^{1}$ Corresponding author.

${ }^{2}$ Present address: Max Planck Institut für Entwicklungsbiologie, D-7400 Tübingen, Germany.
}

tif related to those in the Drosophila Antennapedia and Bithorax homeotic complexes [ANT-C/BX-C (HOM-C)] (Akam 1989). Detailed structural comparisons reveal that the mouse Hox and Drosophila HOM-C share many conserved features, suggesting that they are truly homologous clusters derived from a common ancestor and that they have some conserved functional roles in development (Akam 1989; Duboule and Dollé 1989; Graham et al. 1989; Wilkinson et al. 1989). By analogy to their Drosophila counterparts the vertebrate Hox proteins are believed to function as transcription factors involved in the specification of positional information along the anteroposterior axis. In situ hybridization analyses of $\mathrm{Hox}$ expression patterns during embryogenesis provide strong support for this idea. Spatially restricted, partially overlapping domains of $H O x$ expression are observed in a number of embryonic contexts: the central and peripheral nervous systems, neural crest, somitic mesoderm (prevertebrae), limb, gut, and organs derived from lateral plate mesoderm (Dony and Gruss 1987; Gaunt 1988; Gaunt et al. 1988; Graham et al. 1988a, 1989; Holland and Hogan 1988; Oliver et al. 1988; Dollé and Duboule 1989; Dollé et al. 1989; Dressler and Gruss 1989; Wilkinson et al. 1989; Hunt et al. 1991a,b). These restricted 
patterns of expression are thought to provide part of a basic molecular combinatorial code to specify different regional identities. Experimental support for this hypothesis is derived from null mutants /Chisaka and Capecchi 1991; Lufkin et al. 1991) and from ectopic Hox expression in and retinoic acid treatment of both Xenopus and mouse embryos (Wright et al. 1989; Kessel et al. 1990; Kessel and Gruss 1991; Papalopulu et al. 1991a,b), which result in new combinations of Hox gene products and the transformation or alteration of axial structures. Therefore, understanding the molecular basis of the transcriptional control of the Hox genes is important to examine their function and to identify signals in the regulatory hierarchy.

One of the most striking features of both the Hox and HOM-C gene clusters is that there is a direct correlation between the position of a gene in the complex and its relative domain of expression along the embryonic axis, which is termed spatial colinearity (Lewis 1978; Akam 1987; Gaunt et al. 1988; Dressler and Gruss 1989; Duboule and Dollé 1989; Giampaolo et al. 1989; Graham et al. 1989; Wilkinson et al. 1989). Genes in the Hox clusters all have the same orientation with respect to transcription. Members at the $5^{\prime}$ end are expressed in the more posterior domains and each gene that is successively more $3^{\prime}$ along a cluster has a more anterior boundary of expression. Colinearity has also been observed with respect to the timing of Hox gene activation during embryogenesis (Izpisua-Belmonte et al. 1991) and to the differential sensitivity to retinoic acid in cell lines and embryos of several species, such that the $3^{\prime}$ genes are activated first and are most responsive to retinoic acid (Papalopulu et al. 1990, 1991; Simeone et al. 1990, 1991; Krumlauf et al. 1991). These colinear relationships are highly conserved in all vertebrates examined and further suggest that the Hox complexes act as a molecular representation of different axial coordinates in the embryo.

This extraordinary conservation of structure and function has been interpreted as indicating that the organization of the entire cluster is required for proper regulation and expression. In mice, however, a Hox-1.1-lacZ transgene can be expressed in a normal pattern under the influence of multiple elements that modulate lineagerestricted expression (Puschel et al. 1990, 1991). Similar transgenic studies with other Hox genes reproduce very limited subsets of their proper spatial and temporal patterns (Zakany et al. 1988; Bieberich et al. 1990; Kress et al. 1990; Tuggle et al. 1990). This could be because elements scattered throughout the cluster each regulate either single or multiple genes, or, in the extreme case, because elements exist analogous to the globin locus control region (Grosveld et al. 1987) that impose proper regulation on all the genes. In Drosophila the ANT-C and BX-C are split, whereas in another insect (Tribolium) they are linked (Stuart et al. 1991), and experiments have shown that all of the sequences of the BX-C do not need to be contiguous to rescue mutants in it (Struhl 1984; Tiong et al. 1987). These observations show that some isolated fragments containing several genes of the HOM-C clusters can function normally, although they do not rule out shared regulatory regions between homeotic genes. The presence of multiple transcripts, promoters, splice sites, poly(A) sites, and shared exons in vertebrate Hox genes suggests complex transcription patterns arising from dispersed regulatory regions in the clusters.

In this study we have examined the requirements for the appropriate spatial and temporal regulation of one member of the Hox-2 complex (Hox-2.6) to approach the molecular mechanisms for restricted domains of expression and to provide tools to identify signaling molecules in the regulatory cascade. Using a Hox-2.6-lacZ reporter gene we have successfully reconstructed the major components of the endogenous Hox-2.6 expression pattern on the basis of (1) the proper tissue types, (2) the timing, and (3) spatial restriction. Deletion analysis defined at least three regions involved in the regulation of the gene, two of which had the properties of spatially specific and tissue-specific enhancers. These regions were capable of imposing restricted expression on heterologous promoters and establishing precise boundaries of expression.

\section{Results}

Hox-2.6 is correctly expressed when removed from the cluster

Our goal was to determine whether it is possible to generate the normal pattern of expression of a gene in the Hox-2 complex in transgenic mice. To maximize the chances of obtaining this pattern, initially we used $17 \mathrm{~kb}$ of genomic DNA encompassing the Hox-2.6 gene and its $5^{\prime}$ - and $3^{\prime}$-flanking sequences. Into this we inserted the Escherichia coli lacZ gene in frame in the first exon; this fusion gene directs the synthesis of a protein containing the first 12 amino acids of Hox-2.6 and all of $\beta$-galactosidase (see Fig. 1).

This construct (1) was used to generate transgenic mice that were examined either by transient analysis of $F_{0}$ embryos or by the establishment of lines that were subsequently mated to obtain staged embryos (Table 1 ). All of the lines (5) and transient embryos (17) that expressed the transgene did so in an identical pattern which, in whole-mount staining, resembled that of the endogenous Hox-2.6 gene (Fig. 2). We therefore examined transgene expression in more detail to compare it with the endogenous Hox-2.6 expression pattern determined previously by in situ and Northern analyses.

Hox-2.6 transcripts are normally present in the spinal cord, the hindbrain up to an anterior boundary at the rhombomere $6 / 7$ (r6/7) junction, the cranial and spinal ganglia, the somites and their derivatives with an anterior boundary at prevertebra 2 , and mesodermal components of the lung, gut, esophagus, kidneys, adrenal gland, and gonads (Graham et al. 1988b; Gaunt et al. 1989; Wilkinson et al. 1989; Graham 1990). Our analysis of whole-mount staining clearly showed that there was expression in all of the appropriate tissues (Fig. 2). This was observed most clearly in the staining of viscera from a 14.5-day postcoitum (dpc) transgenic embryo that showed no expression in the thyroid, heart, or liver but 

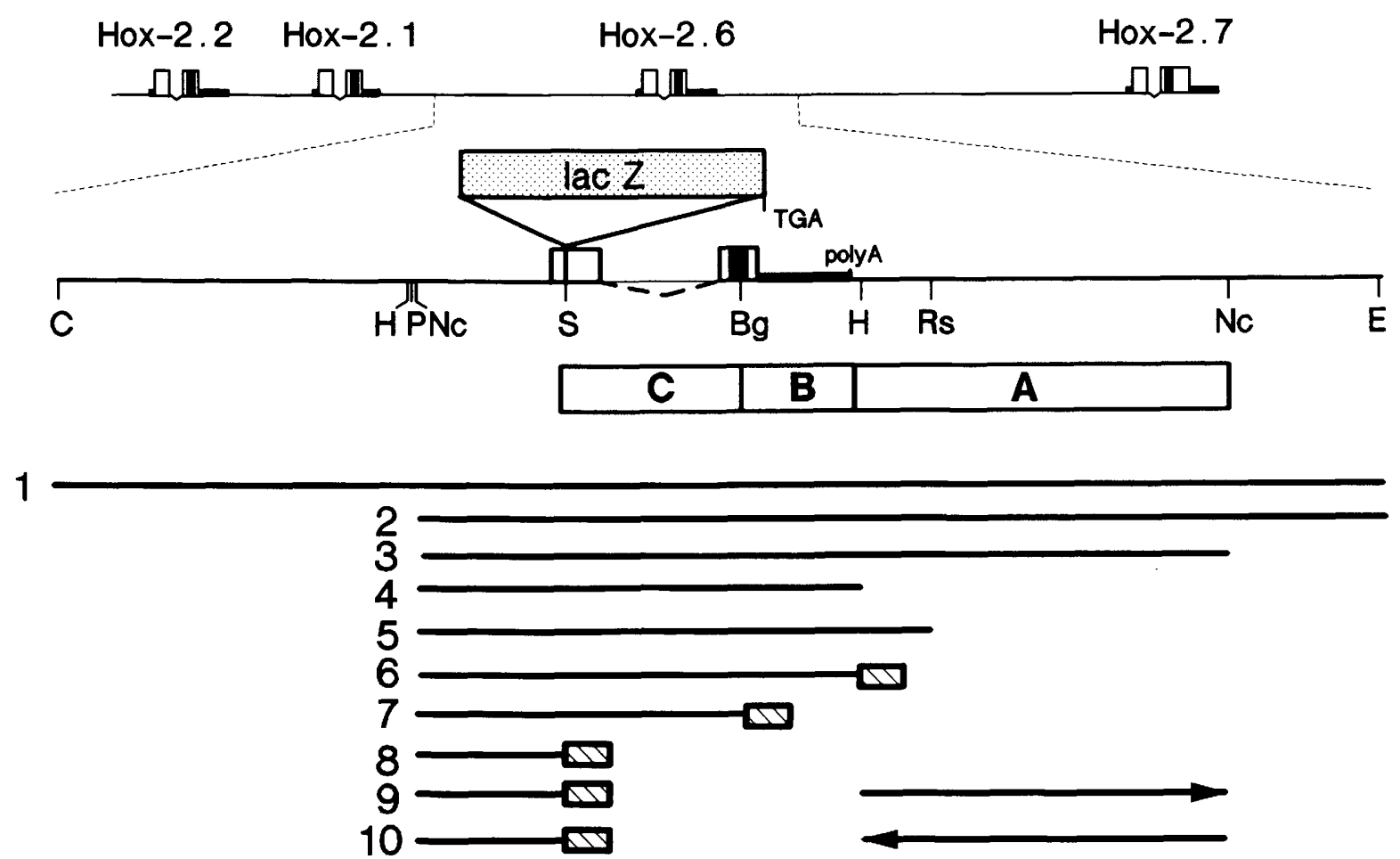

Figure 1. Region surrounding the Hox-2.6 gene showing an enlargement of the gene structure and the deletions used in transgenic mice. The enlargement shows the restriction sites used to generate the deletions: (C) ClaI; $(\mathrm{H})$ HindIII; $(\mathrm{P})$ PstI; (Nc) NcoI; (S) SalI; (Bg) $B g I I I ;$ (Rs) RsaI; (E) EcoRI. (The diagram is not to scale.) The stippled region represents the lacZ gene that has been inserted in-frame into the first exon of Hox-2.6 at the SalI site. The boxes labeled A, B, and C represent the regulatory regions described in the text. The deletion fragments shown in the lower part align with the restriction sites above. The hatched boxes on the $3^{\prime}$ ends of constructs $6-10$ represent the SV40 polyadenylation signal. The arrows in constructs 9 and 10 show the $5^{\prime} \rightarrow 3^{\prime}$ orientation of region A in the two constructs.

did show expression in the gut, metanephric kidney, mesonephros and adrenal glands, hair follicles, and mammary glands, and weak expression in the gonads. In only one case did we observe a difference between the transgenic and endogenous Hox-2.6 patterns. The endogenous gene was expressed throughout the lung, but the transgene (both protein and RNA) was expressed in posterior parts of the largest lobes of the lung up to a distinct anterior boundary that did not correlate with any obvious morphological feature.

We then determined whether the spatial distribution was correct by examining the boundaries of expression in several tissues. At 9.5-10.5 dpc rhombomeres are morphologically identifiable, and a sharp limit of staining was observed at the $\mathrm{r} 6 / 7$ boundary, just posterior to the otic vesicle (Fig. $2 \mathrm{~d}$ ), which is the proper anterior limit of expression in the hindbrain. With respect to para-axial mesoderm, histological analysis of prevertebrae revealed strong staining of PV3 and more posterior prevertebrae and some positive cells in PV2 (Fig. 2e). This agrees with the boundary of expression of the endogenous gene mapped to PV2 by in situ hybridization (Fig. 2f; Gaunt et al. 1989). At $12.5 \mathrm{dpc}$ the Hox-2 genes display sharp dorsally restricted expression in the spinal cord (Graham et al. 1991), and transverse sections showed that the transgene also respected the appropriate dorsoventral limits. We noted high levels of staining in the dermal placodes of developing hair follicles and mammary glands, and there was a clear axial limit to the follicle expression. Endogenous expression of Hox2.6 has not been examined in skin but we feel that this spatial restriction is likely to reflect an aspect of the normal pattern in light of the correspondence in other tissues.

To examine temporal regulation the transgenic lines were used to produce staged embryos from 8.5 to 14.5 dpc. In the 8.5 -dpc embryo the staining was weak but clearly present in the neuroectoderm, somites, and lateral plate mesoderm from the posterior end to an anterior boundary consistent with that seen by in situ analysis (Fig. 2i). At $9.5 \mathrm{dpc}$ the rhombomeres had formed and neural expression was clearly at its correct $\mathrm{r} 6 / 7$ boundary (Fig. 2j). Expression was maintained in all of the correct tissues until $14.5 \mathrm{dpc}$, which is the latest stage convenient for whole-mount staining. We have also examined dissected tissues from neonatal and adult animals and found that the transgene is appropriately expressed, by comparison with previous Northern analyses (Graham et al. 1988b, 1989).

Whole-mount staining examines the distribution of $\beta$-galactosidase, whereas the determination of the normal pattern was done at the level of mRNA. In situ anal- 
Table 1. Transgenic mice examined

\begin{tabular}{|c|c|c|c|c|c|}
\hline \multirow[b]{2}{*}{ Construct $^{\mathrm{a}}$} & \multirow[b]{2}{*}{ Assay } & \multicolumn{4}{|c|}{$\begin{array}{l}\text { Transgenic expression patterns } \\
\text { compared to endogenous } \\
\text { Hox- } 2.6 \text { pattern }\end{array}$} \\
\hline & & $\begin{array}{l}\text { total } \\
\text { no } \exp ^{\mathrm{b}}\end{array}$ & complete & $\begin{array}{l}\text { consistent } \\
\text { subset }\end{array}$ & ectopic \\
\hline \multirow[t]{2}{*}{1} & transient & $17 / 18$ & 17 & - & - \\
\hline & lines & $5 / 5$ & 5 & - & - \\
\hline \multirow[t]{2}{*}{2} & transient & $2 / 3$ & 2 & - & - \\
\hline & lines & $1 / 2$ & 1 & - & - \\
\hline \multirow[t]{2}{*}{3} & transient & $6 / 10$ & 6 & - & - \\
\hline & lines & $2 / 5$ & 2 & - & - \\
\hline \multirow[t]{2}{*}{4} & transient & $0 / 8$ & - & - & - \\
\hline & lines & - & - & - & - \\
\hline \multirow[t]{2}{*}{5} & transient & $3 / 3$ & - & 3 & 1 \\
\hline & lines & - & - & - & - \\
\hline \multirow[t]{2}{*}{6} & transient & $5 / 8$ & - & 4 & 1 \\
\hline & lines & $4 / 4$ & - & 2 & 2 \\
\hline \multirow[t]{2}{*}{7} & transient & 11 & - & 11 & - \\
\hline & lines & $6 / 12$ & - & 3 & 3 \\
\hline \multirow[t]{2}{*}{8} & transient & $5 / 8$ & - & - & 5 \\
\hline & lines & $3 / 7$ & - & - & 3 \\
\hline \multirow[t]{2}{*}{9} & transient & $4 / 4$ & - & 4 & 1 \\
\hline & lines & $4 / 5$ & - & 3 & 1 \\
\hline 10 & transient & $1 / 1$ & - & 1 & - \\
\hline 11 & transient & 3 & - & 3 & 1 \\
\hline 12 & transient & 8 & - & 8 & 5 \\
\hline 13 & transient & 3 & - & 3 & 3 \\
\hline
\end{tabular}

${ }^{a}$ The construct numbers correspond to those used in Figs. 1 and 4.

b/Total no exp) The number of positively stained lines or embryos as a fraction of the total number of transgenic mice. In transient assays the number of transgenic embryos was not always determined, and the amount shown indicates the number that were positively stained. The types of patterns observed are defined in the text. The numbers in the last two columns may add up to more than the number shown in this column, which indicates that some embryos showed ectopic patterns of expression in addition to a consistent subset of the normal pattern.

ysis with lacZ and Hox-2.6 probes allowed a direct comparison between the distributions of these two mRNAs and showed that they were indistinguishable in all respects apart from in the lung. In particular, the boundaries of the lacZ mRNA in the hindbrain and prevertebrae were correct (Fig. 2f-h). The specific activities of the two probes were similar, as were the exposure times, indicating that in addition to the proper spatial and temporal regulation the levels of expression of the transgene and the endogenous gene were comparable.

These results led us to conclude that the overall pattern of Hox-2.6 expression does not require the gene to be located within the cluster and that the $17-\mathrm{kb}$ fragment contains those elements necessary for correct expression in terms of tissue specificity, timing, and spatial localization. However, our analysis cannot exclude minor deviations from the normal pattern.

\section{Deletion analysis delineates three regulatory regions}

Having substantially reconstructed the expression pattern of Hox-2.6, deletion analysis was carried out to de- termine whether the pattern could be broken down into component parts and, if so, to delineate the regions responsible. Figure 1 shows the constructs examined and Table 1 shows the numbers of transgenic animals obtained with a general description of the patterns observed. We distinguish between the normal expression pattern described above, which we define as complete, consistent subsets of the complete pattern, and patterns that vary from embryo to embryo or are inappropriate, which we define as ectopic.

The first two deletions ( 2 and 3 ) gave the same expression pattern as construct 1 showing that only $7.4 \mathrm{~kb}$ encompassing the Hox-2.6 gene is required for proper expression (Fig. 3). The next series of deletions defined three regions important for aspects of Hox-2.6 expression. Construct 4 did not express, and as it ends just $3^{\prime}$ of the AATAAA sequence of Hox-2.6 it was possible that polyadenylation of the transgene mRNA was abnormal. To overcome this problem an SV40 polyadenylation signal was added to the $3^{\prime}$ end, and in transgenic mice the majority of the expression pattern was normal but there was a clear caudal shift in the anterior boundary of expression in the central nervous system (CNS) (Fig. 3c). Expression from this construct (6) appeared to terminate, not at the correct $\mathrm{r} 6 / 7$ limit, but abruptly between the spinal cord and the hindbrain. This more caudal boundary appeared to correspond to that of normal Hox-2.1 expression (Graham et al. 1989; Wilkinson et al. 1989), suggesting that the deleted region is necessary for setting the correct anterior boundary of Hox-2.6 expression in the hindbrain. In all other respects the expression of the $1 a c Z$ reporter gene appeared normal (Fig. 3). To ensure that the SV40 sequences were not responsible for this changed expression pattern, another version was made (5, Fig. 1) extending $3^{\prime}$ sequences 700 bp past the normal poly(A) site. It gave an expression pattern identical to that observed with the SV40 sequences, showing that they did not perturb the expression pattern.

In the next deletion the $3^{\prime}$-untranslated region and part of the second exon were removed and replaced with an SV40 polyadenylation signal $(7$, Fig. 1). The only detectable difference in transgenic mice carrying this construct is a complete absence of expression in the lung (Fig. 3d), implicating the deleted region $(B)$ as necessary for part of the expression in this tissue.

Finally, a deletion $(8)$ that leaves only 5 '-flanking sequences and the first 12 codons was tested, and it gave a random distribution of expression (Fig. 3e), presumably owing to sensitivity of the 5 -flanking sequences to regulatory regions around the integration site. This result demonstrated that the 5 -flanking sequences are capable of directing expression but incapable of imposing any aspect of the normal spatial pattern (Fig. 3). One site of expression that occurred frequently in embryos bearing construct 8 is a region of the midbrain corresponding to superficial and lateral regions of the superior colliculi. This staining is also seen in mice with constructs 6 and 7 , in addition to the subsets of the normal pattern; although the significance of expression in this region is not clear, it could indicate that constructs 1-3 contain infor- 
Whiting et al.

mation that prevents Hox-2.6 expression in this part of the midbrain.

This deletion analysis has defined three regions $(A-C)$ important in forming the pattern of Hox-2.6 expression (see Fig. 1). Region A is defined by the $3-\mathrm{kb}$ fragment immediately $3^{\prime}$ of the polyadenylation site and appears to be necessary for the establishment of the correct anterior boundary of expression in the central nervous system (CNS). Region B contains the exon 2 sequences encoding the last 44 amino acids of the protein and the 3 '-untranslated sequences and is necessary for expres- sion in the lung. The majority of the pattern requires region $\mathrm{C}$, which includes the intron and flanking exon sequences. With respect to the promoter, the $1.3 \mathrm{~kb} 5^{\prime}$ of the ATG may also contain elements important in specifying the pattern that cannot function in the absence of other signals.

\section{Region A contains a neural enhancer capable of setting the anterior boundary}

We examined region A further in the absence of regions

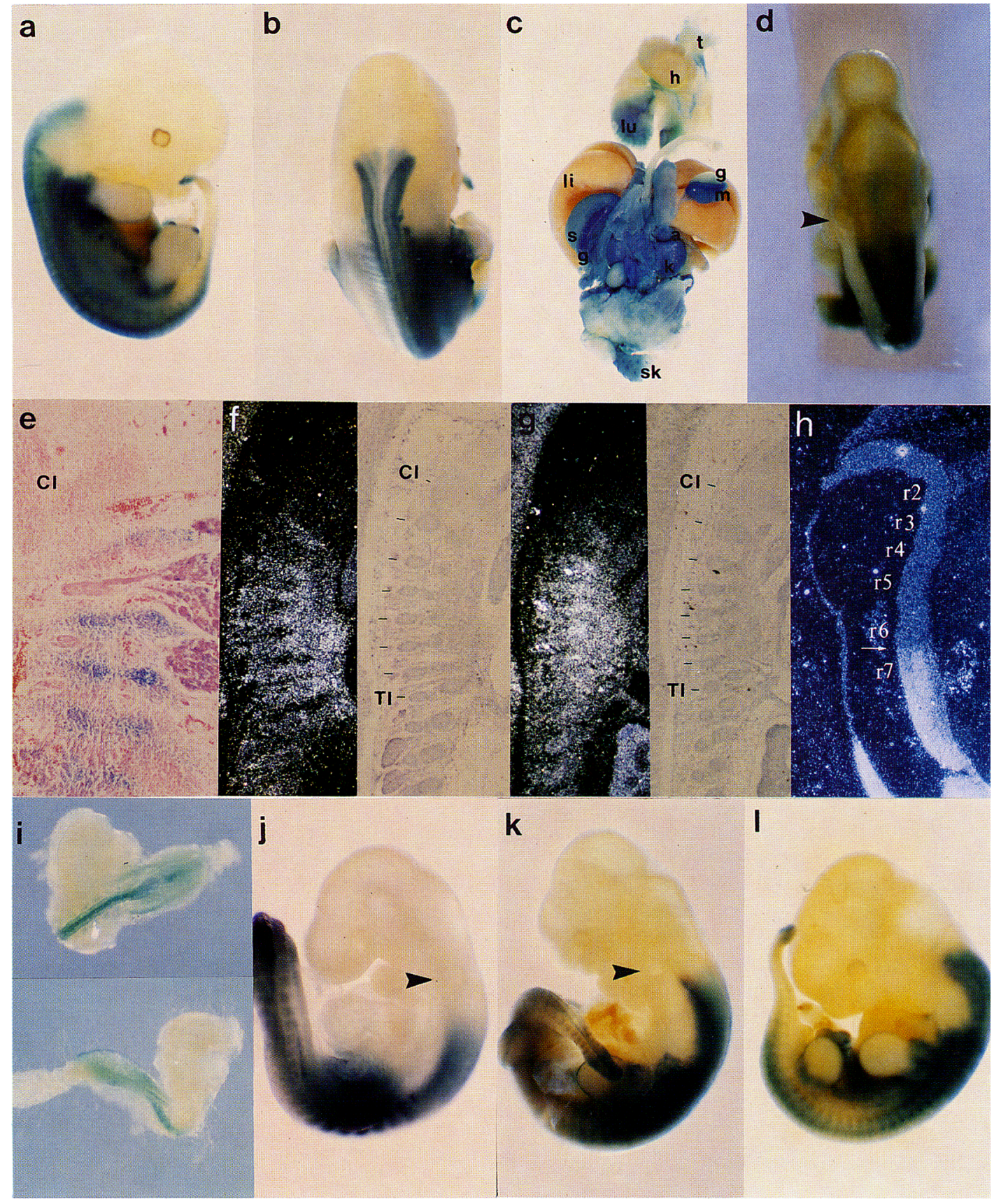

Figure 2. (See facing page for legend.) 


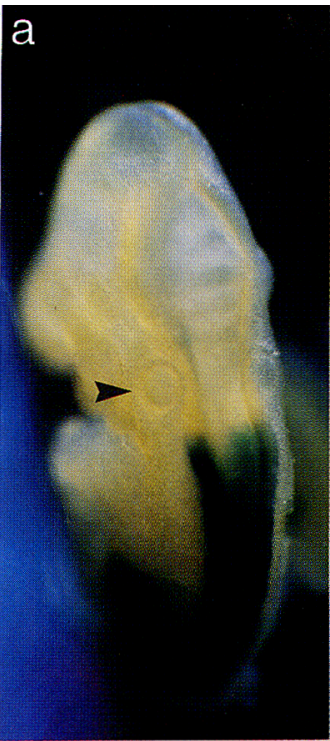

f

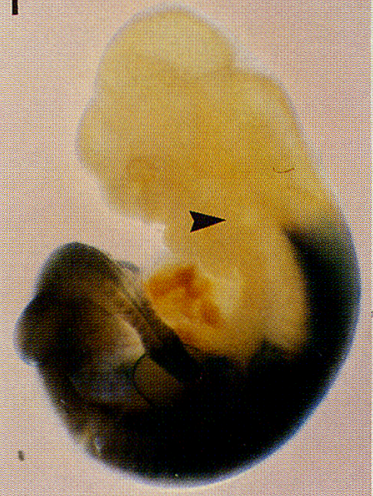

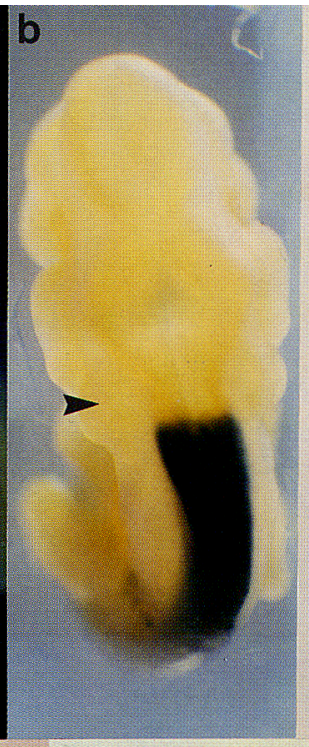

g

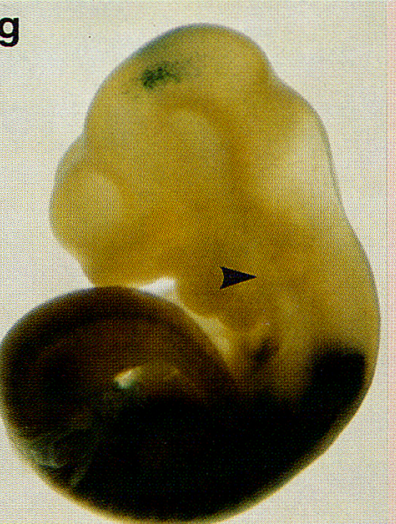

c

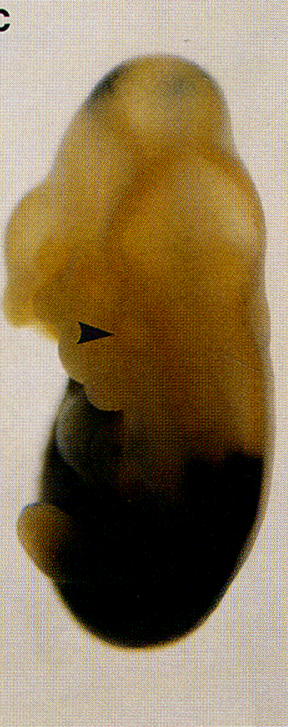

h

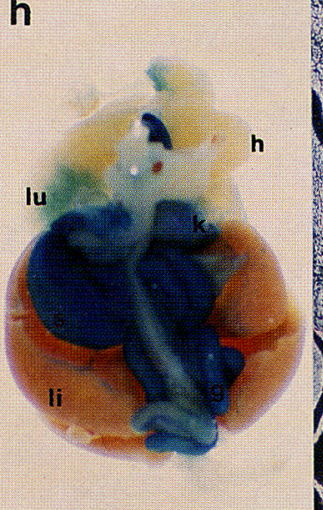

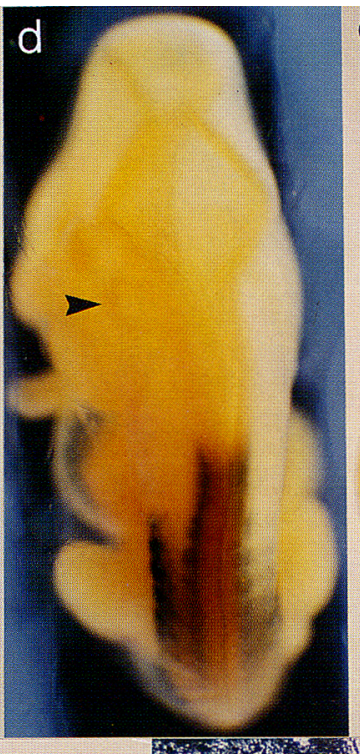

e

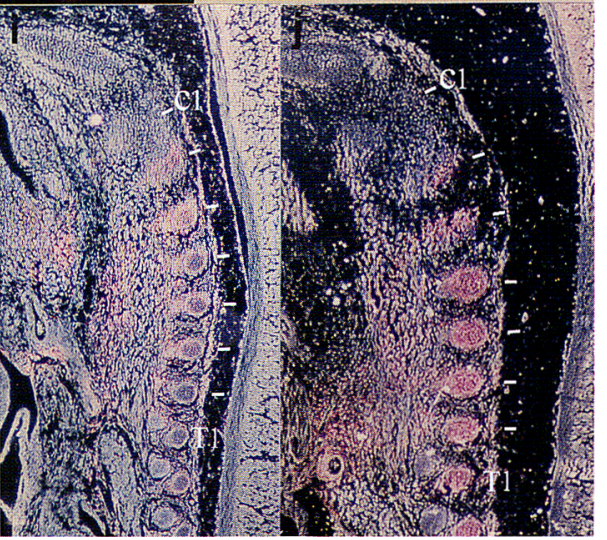

Figure 3. Transgenic 10.5-dpc embryos stained for $\beta$-galactosidase activity showing the effects of the different deletion constructs on the expression pattern. $(a)$ Construct $2 ;(b)$ construct $3 ;(c)$ construct $6 ;(d)$ construct $7 ;(e)$ construct $8 ;(f)$ construct $1 ;(g)$ construct 6 . The otic vesicle sits between the boundary of rhombomeres 5 and 6 and is marked with an arrowhead. (h) Viscera from a 14.5-dpc transgenic embryo carrying construct 6 showing that the pattern in the internal organs is the same as with construct 1 . The labeling is the same as in Fig. 2. (i-j) Dark-field pictures of sectioned transgenic embryos lightly counterstained with eosin. Under dark-field illumination the blue-stained regions appear pink. $i$ carries construct $3 ; j$ carries construct 7 . These sections show a boundary of expression in the prevertebrae in PV2. The first cervical and first thoracic prevertebrae are labeled accordingly.

B and C by placing it immediately $3^{\prime}$ of the SV40 polyadenylation signal of construct 8 (Figs. 1 and 4 ). This tests the ability of this region to regenerate the compo- nent part of the pattern that was lost with its deletion. We observed the correct anterior limit of expression in the hindbrain at $\mathrm{r} 6 / 7$ irrespective of the orientation of

Figure 2. Expression of construct 1 resembles that of the endogenous Hox-2.6 gene. (a) Lateral view of a 12.5-dpc transgenic embryo stained for $\beta$-galactosidase activity. Expression can be seen in the hindbrain, spinal cord, spinal ganglia, and prevertebrae. $(b)$ Dorsal view of the same embryo. (c) Viscera from a 14.5-dpc transgenic embryo showing staining in the stomach $(\mathrm{s})$, gut $(\mathrm{g})$, metanephric kidneys $(\mathrm{k})$, adrenal gland $(\mathrm{a})$, mesonephric tubules $(\mathrm{m})$, lung $(\mathrm{lu})$, hair follicles in the skin (sk), and connective tissue, and slight staining in the gonad (go). There is no staining in the thymus ( $\mathrm{t})$, heart (h), liver (li), or anterior lung. (d) Dorsal view of a 10.5-dpc transgenic embryo showing the anterior limit of staining in the hindbrain at the r6/7 boundary. The otic vesicle sits between rhombomeres 5 and 6 and serves as a positional marker for rhombomeres in the hindbrain. (e) Sagittal section of a 12.5-dpc transgenic embryo counterstained with eosin showing prevertebrae and spinal ganglia with the limit of staining at PV2. The first cervical prevertebra is labeled C1. (f) Dark-field illumination of in situ hybridization with a lac $Z$ probe to a sagittal section of a transgenic 12.5-dpc embryo adjacent to bright-field illumination of the same section showing an anterior boundary of hybridization to PV2. The first cervical and first thoracic prevertebrae are labeled accordingly. $|g|$ Near adjacent section hybridized with a Hox-2.6 probe showing the same pattern as with the lac $Z$ probe. In $f$ and $g$ the anterior limit of staining is in C2. (h) Dark-field image of an in situ hybridization with the lacZ probe to a sagittal section of a transgenic 10.5-dpc embryo showing an anterior boundary of expression to the $\mathrm{r} 6 / 7$ boundary in the hindbrain. (i-1) Time course showing the staining patterns obtained with construct 1 in embryos 8.5, 9.5, 10.5, and 11.5 dpc. The arrowheads show the position of the otic vesicle where it is visible in the younger embryos. 
region A (Fig. 5). However, staining was also observed along the full length of the spinal cord in a domain not perturbed by the deletion of region A. It was also seen in the developing spinal ganglia in 10.5-dpc embryos. That region A can drive expression throughout the spinal cord suggests that some aspects of the Hox-2.6 pattern may be mediated by redundant elements. This experiment showed that region A is capable of interacting in an orientation-independent manner with its own promoter to specify the formation of the normal rhombomere limit of neural expression.

\section{Regions $A$ and $C$ impose spatial specificity on heterologous promoters}

We wished to determine whether the positional specificity resides in regions $A$ and $C$ alone. Initially we examined the ability of region A to interact with the promoter of Hox-2.1, another gene in the Hox-2 complex that has a more posterior expression domain. In construct 11 (Fig. 4) region A was placed 3' of a 5.5-kb fragment of Hox-2.1 DNA that had the lacZ gene inserted into the first exon. This basic Hox-2.1 clone is only capable of low-level mesodermal expression and barely detectable neural tube expression (S. Nonchev and R. Krumlauf, unpubl.). The effect of region A on Hox-2.1 expression was striking in that there was strong expression in the neural tube up to the Hox-2.6 boundary at $r 6 / 7$, the same pattern as seen with region A on its own promoter (Fig. 5). The remainder of the staining pattern was that seen with the Hox-2.1 base construct. The presence of the Hox-2.6, as opposed to the Hox-2.1, rhombomere boundary shows that the specificity resides in the region A enhancer rather than in the Hox-2.6 promoter.

To investigate the ability of region A to confer spatial specificity to a non-Hox heterologous promoter, it was

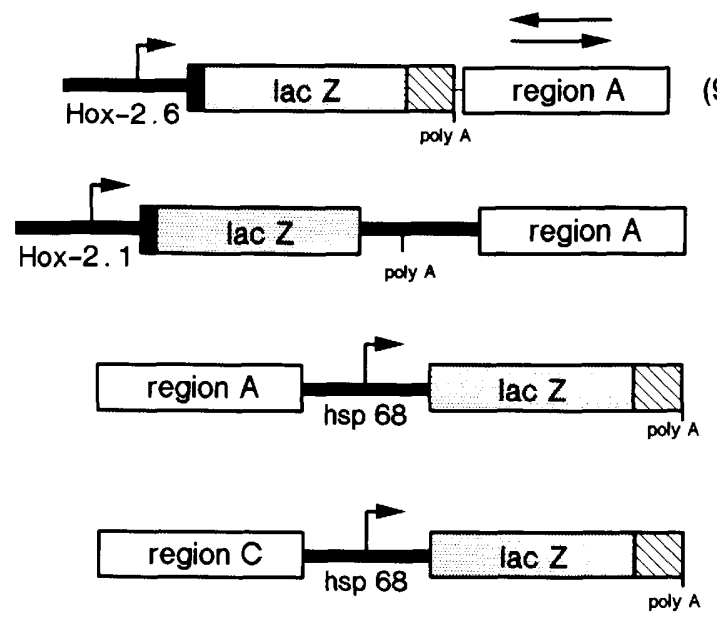

Figure 4. Constructs for enhancer analysis. Constructs 9 and 10 are as shown in Fig. 1. Solid black lines represent the appropriate genomic DNA; stippled boxes represent the coding region of the lacZ gene; striped boxes denote the SV40 polyadenylation signal; open boxes show the regulatory regions described in the text. The bent arrows indicate the start of transcription in the different constructs. juxtaposed to the $h s p 68$ minimal promoter immediately $5^{\prime}$ of the lacZ gene (12, Fig. 4). The expression pattern was the same as that observed with constructs 9 and 10 , that is, expression was confined to the neural tube up to the normal Hox-2.6 boundary at $\mathrm{r} 6 / 7$. This demonstrates that region A contains enhancers capable of imposing their spatial specificity on heterologous promoters.

In an analogous manner, region $\mathrm{C}$ was placed adjacent to the $h s p 68$ promoter $(13$, Fig. 4$)$ to determine whether it behaved as a spatially specific enhancer, capable of directing expression in the major remaining domain of the Hox-2.6 pattern. The expression pattern had two components, the first very similar to that seen when region $C$ interacts with its own promoter (construct 7 ), with staining in the spinal cord to the boundary with the hindbrain, spinal ganglia, somites, and developing visceral organs. The second, weaker component observed on longer staining, comprises ectopic domains in the more anterior CNS that were not seen with any of the other clones. This may reflect the interaction of region $\mathrm{C}$ with this particular promoter. However, it is clear that region $C$ has the ability to impose the same pattern of expression on its own and heterologous promoters.

\section{Discussion}

The approach we have taken to investigate the regulation of the Hox-2.6 gene has involved the use of transgenic mice carrying fusion genes containing the $E$. coli lacZ gene as a reporter. Using this method we have reconstructed major components of the normal pattern of Hox-2.6 expression outside of the cluster. We have subsequently been able to identify elements that regulate this pattern, some of which were then shown to act as spatially specific enhancers on heterologous promoters. These elements will allow us to study the response of the Hox genes to upstream signaling information and the means by which it is interpreted to bring about the final complex patterning of the embryo.

An important advantage of having reconstructed the endogenous Hox-2.6 pattern is that, in the absence of antibodies, the transgenic mice will allow a more detailed description of the cell types that normally express the gene. For example, we detected expression in the dermal placodes of the developing hair follicles, which had not been identified as sites of Hox-2.6 expression by in situ analysis. On the basis of stringent criteria used to define the normal pattern and the fact that no other additional sites of transgene expression were detected compared with the endogenous gene, we think it likely that this reflects a genuine aspect of Hox-2.6 expression. Mice marked in this way will also be extremely useful for a variety of lineage tracing and grafting studies to follow developmental interactions in embryonic structures.

Clustered organization is not required for Hox-2.6 expression

We have shown that the normal position of the Hox-2.6 gene, in the heart of the cluster, is not necessary for 


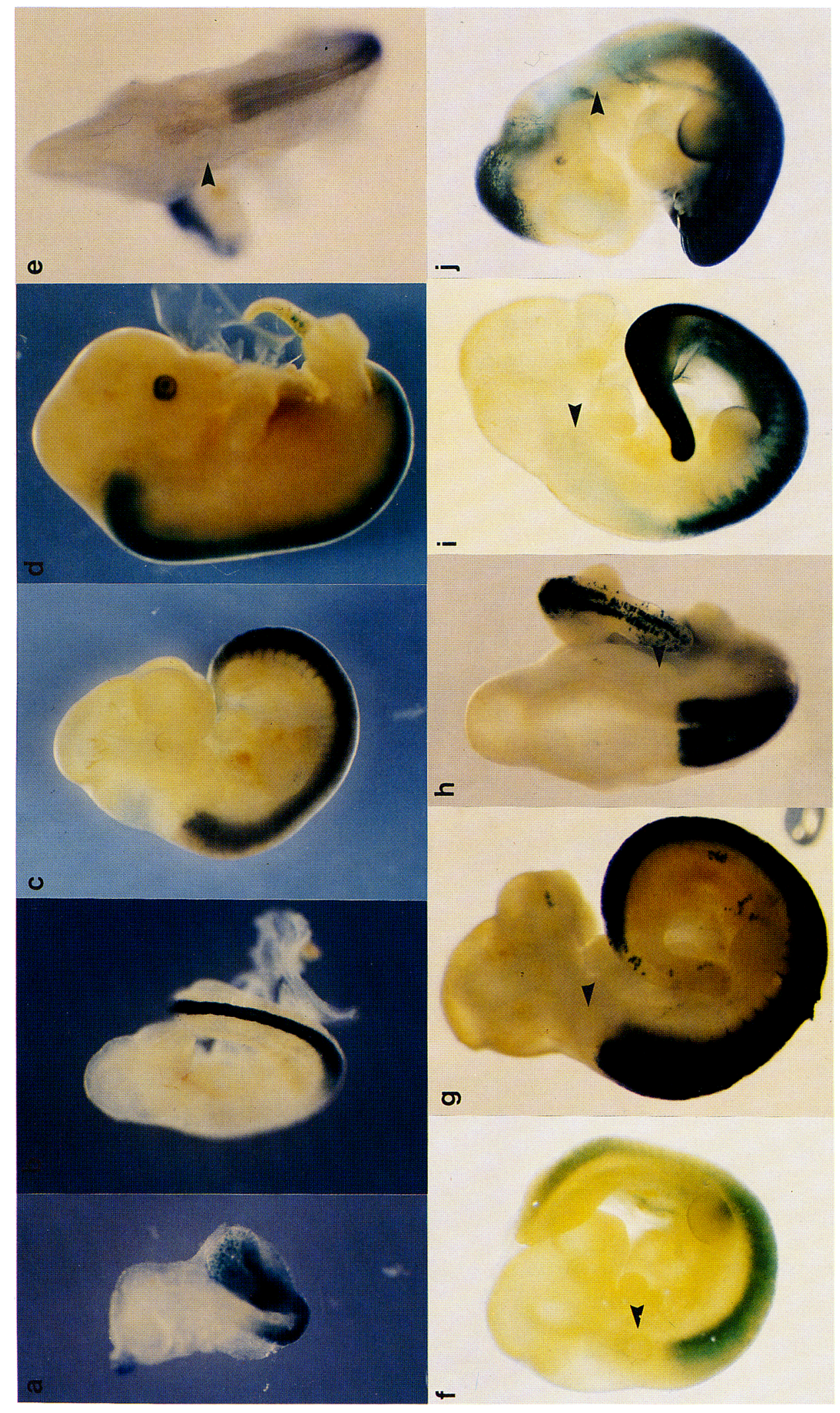

它芯芯芯

중요요

舟䓽

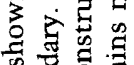

a 영

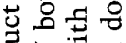

政.

:

0 政

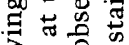

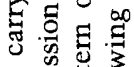

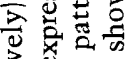

岁. 这

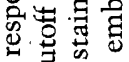

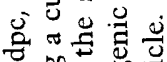

i⿱宀⿻三丨口

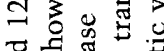

उ क्ष̃

กิ

열

เก

更

我

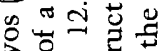

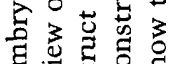

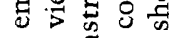

㟧范

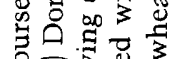

ठ․

官

렬

I

함 跣

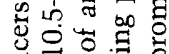

击

प्य

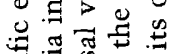

可

की

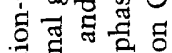

责言要

品尊

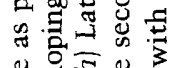

遂质

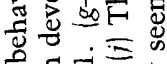

$0 . \Xi \pm \div$

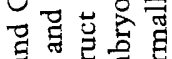
《告要

象

엉

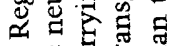
ค 웡 เ⿵

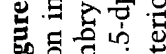

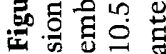


expression. Mice carrying the largest construct encompassing the gene and its immediate flanking sequences $(17 \mathrm{~kb})$ have both lacZ mRNA and $\beta$-galactosidase present in a pattern nearly indistinguishable from that of Hox-2.6 mRNA. With the exception of regionalized expression in the lung, the embryos show the correct anteroposterior and dorsoventral boundaries of expression, the correct tissue distribution, and the proper temporal progression. Furthermore, as judged by in situ analysis, the levels of expression are comparable with those of the endogenous gene.

\section{Hox-2.6 is regulated by spatially specific enhancers}

The deletion analysis detailed in this study identified three regulatory regions, $\mathrm{A}, \mathrm{B}$, and $\mathrm{C}$. Region $\mathrm{A}$ is a $3-\mathrm{kb}$ fragment $3^{\prime}$ of the polyadenylation site that generated expression in the neural tube with a sharp anterior boundary in the hindbrain at $\mathrm{r} 6 / 7$. It functioned in both orientations on the Hox-2.6 promoter and was also capable of imposing neurally restricted expression with the appropriate anterior boundary on the heterologous Hox2.1 and $h s p 68$ promoters. More extensive analysis will reveal whether a single element is responsible for both tissue and spatial restrictions. The lacZ expression seen in the superior colliculi with constructs lacking region A could be interpreted as suggesting that a negative regulatory element is present within this $3 \mathrm{~kb}$ that, under normal circumstances, prevents Hox-2.6 expression in that area.

Region B was identified as being necessary for part of the endogenous expression in the lung but has not yet been analyzed in detail. Because Hox-2.6 is normally expressed throughout the lung, it appears that multiple elements are necessary to generate the complete lung pattern. Expression only in the more posterior regions of the lung suggests that region $B$ is not simply a tissue-specific element but that it may also set spatial boundaries. This serves to indicate that spatial as well as tissue-specific information is likely to be involved in generating expression patterns in the visceral mesoderm as well as in the axial mesoderm and neural tube.

Region $\mathrm{C}$ contains the intron and parts of the two flanking exons and is responsible for expression in the majority of the neural and mesodermal domains. This region is also able to function outside of the transcription unit to impose an identical pattern on the heterologous hsp 68 promoter. Taken together, these data demonstrate that the information for directing regional specificity resides in regions $\mathrm{A}$ and $\mathrm{C}$ and not in the promoter itself, and that both of these regions are capable of acting as spatially specific enhancers.

Neural expression is not specified by a unique element, as both regions $A$ and $C$ work independently to drive expression in the spinal cord. A potential redundancy therefore exists among the Hox-2.6 regulatory elements, although we have not examined in detail whether the timing and the dorsoventral distribution of expression are identical in the two spinal cord patterns. The anteroposterior domain of expression in the spinal cord generated by region $\mathrm{C}$ appears to be that of the Hox2.1 gene; one explanation for this apparent redundancy may be that region $\mathrm{C}$ contains neural control elements that normally act on the adjacent Hox-2.1 gene.

Many transcripts varying from 2.4 to $10 \mathrm{~kb}$ and multiple starts have been observed for the Hox-2.6 gene, but it is not known how many promoters are used to drive this expression (Graham et al. 1988b). Hox-2.6 in situ analysis used a common exon probe and would not have distinguished between these different transcripts or revealed any spatial or temporal differences caused by differential promoter utilization. It is possible, therefore, that elements exist in addition to the three we have identified that are necessary to give the correct balance of expression from all the promoters normally used.

Our experiments do not define the nature of the factors that interact with the cis-acting regulatory regions identified in this study. The Hox-2.6 transgene was always examined in the presence of the wild-type protein; given that Hox-2.6 is related to the Drosophila Dfd gene, which is known to be regulated by its own product (Bergson and McGinnis 1990|, one of these factors may be the Hox-2.6 protein itself. However, in Drosophila, autoregulation of the $D f d$ gene only occurs in a small subset of $D f d$ expression domains. The Hox-2.6 enhancers function independently on heterologous promoters to establish the complete pattern of expression, and it is difficult to envisage how autoregulation can be the sole mechanism responsible. It will be possible to test whether autoregulation plays any role in Hox-2.6 expression by examining the expression of the transgene in null mutants and by defining the binding sites required for proper regulation. Despite the general conservation between species, caution should be exercised in the degree to which Drosophila and vertebrate Hox genes are thought to use identical regulatory sequences. We feel that it is likely that homologs in these disparate systems have evolved substantial differences in the type, position, and organization of their regulatory elements.

\section{Conservation of the clustered arrangement and the potential for shared regulatory elements}

Despite the fact that the Hox-2.6 gene functions outside of the complex, the clustered arrangement may nonetheless play a role in the regulation of the Hox-2 genes. Regulatory elements for other Hox-2 genes may also be contained in construct 1. Although Hox-2.6 can be isolated from the cluster, the expression potential of the remaining genes could be compromised by the loss of these elements. In support of this, a small $5^{\prime}$ exon of the adjacent Hox-2.7 gene is present in the center of the $3 \mathrm{~kb}$ of region A, $25 \mathrm{~kb}$ upstream of the Hox-2.7 ATG (M.-H. Sham and R.Krumlauf, unpubl.). It appears that a Hox2.7 promoter also resides in this Hox-2.6 regulatory region, demonstrating that overlapping elements do exist within the Hox-2 cluster.

This leads us to speculate that there are interspersed and/or overlapping regulatory elements in the Hox-2 complex. Therefore, Hox-2.6 may be unusual in that its 
control regions reside within the gene itself and the immediate flanking regions. This is supported by the fact that in extensive transgenic analyses of other genes in the Hox-2 cluster we have failed to reconstruct completely normal patterns of expression. Moreover, other workers have described experiments aimed at reconstructing normal expression patterns of other Hox genes with varying degrees of success. Puschel et al. (1990, 1991) have defined, by deletion analysis, three elements that are required for the establishment and maintenance of the proper pattern of Hox-1.1 expression. As with Hox-2.6, multiple elements are located within and adjacent to the gene but it is clear that the regulatory mechanisms are distinct. In marked contrast to the Hox-2.6 promoter, the Hox-1.1 promoter is ubiquitously active, and this activity is restrained by elements that prevent expression in spatially and temporally restricted domains. Although the experiments of Puschel et al. (1991) do not address the question of whether these elements can act on heterologous promoters, the data available for these two genes show that there is no universal architecture of Hox gene regulatory elements. Other studies describe very limited subsets of the normal expression patterns that have been attributed to the absence of remote regulatory sequences from the constructs tested; in one case, a role for specific enhancers has been suggested (Zakany et al. 1988; Bieberich et al. 1990; Kress et al. 1990; Tuggle et al. 1990).

We therefore believe that a major selective pressure preserving the structures of the homeo box complexes is based on regulatory mechanisms and results from the dispersed location of the regulatory elements that act on individual genes or sets of genes. We do not mean to imply that identical regulatory elements are present in other species but, rather, that some of the regions that are implicated in the regulation of the genes are interspersed, making it difficult to disrupt the complexes.

Having identified major regions capable of regulating Hox-2.6 independently of the cluster, we are now in a position to subdivide regions $\mathrm{A}$ and $\mathrm{C}$ more extensively into their component elements and to examine the trans-acting factors that interact with them, thereby gaining insight into the hierarchies involved in developmental control. The significance of the complex transcription pattern of the endogenous gene will be addressed in experiments in which the elements described here, in conjunction with the Hox-2.6-coding sequences, are used to attempt to rescue null mutants. We will also be able to use the elements to alter selectively the expression domains of the Hox gene and other developmentally important genes and thereby better study their function.

\section{Materials and methods}

\section{DNA manipulations}

Construct 1 was made by first digesting pcosH9 /Graham et al. 1988b) with ClaI, end-filling with Klenow, and digesting with NcoI. This fragment was ligated with the adjoining Ncolpartial)-EcoRI fragment into pPolyIII $\Delta$ SalI [pPolyIII (Lathe et al.
1987) that had been cut with SalI, end-filled with Klenow, and religated to form pPolyIII $\Delta S a l I]$ that had been digested with $S m a I$ and EcoRI. The lacZ gene was inserted in-frame into this plasmid at the SalI site. The SalI ends were filled in with $\mathrm{T}$ and $\mathrm{C}$ and the BamHI ends on the lacZ gene were filled in with $\mathrm{G}$ and $\mathrm{A}$ to give compatible overhangs. The two fragments were ligated to give construct 1 . The insert was excised for injection with NotI. Construct 2 was formed by digesting construct 1 with PstI to prepare injection fragment. Likewise, construct 3 was prepared by digestion with NcoI, and construct 4 with Pst I and HindIII. Construct 5 was made by digesting construct 4 with BamHI, end-filling, digesting with HindIII, and ligating it to a $0.7-\mathrm{kb}$ HindIII-RsaI(end-filled) fragment containing the sequences directly $3^{\prime}$ of the HindIII site of construct 4. Construct 6 was made by ligation of the $6.4-\mathrm{kb}$ PstI-HindIII fragment from construct 1 with the $0.24-\mathrm{kb}$ HindIII-BamHI fragment containing the SV40 polyadenylation sites from pGEMT into BamHIPstI cut pPolyIII-I. pGEMT contains the SV40 polyadenylation sites as a BclI-EcoRI fragment in the BamHI-EcoRI site of pGEMI. Construct 7 was made by ligating the $5.6-\mathrm{kb}$ PstI-BglII fragment from construct 1 with the $0.24-\mathrm{kb}$ BamHI-HindIII fragment of pGEMT into the HindIII-PstI site of pPolyIII-I. Construct 8 uses the $2.1-\mathrm{kb}$ PstI-ClaI fragment from construct 7 containing the $5^{\prime}$ end of $H o x-2.6$ and the first $0.8-\mathrm{kb}$ of the $l a c Z$ gene, and the $2.43-\mathrm{kb}$ ClaI-BamHI fragment from p610ZA (kindly provided by J. Rossant) containing the rest of lacZ and the SV40 polyadenylation sites that were ligated into the PstIBamHI site of pPolyIII-I. Constructs 9 and 10 were made by ligating the end-filled HindIII-NcoI fragment into construct 8, which had been digested with $B a m H I$ and end-filled. Injection fragments for constructs 5-10 were prepared by digestion with NotI. To put region A onto the hsp 68 promoter the end-filled HindIII-NcoI fragment was ligated into the end-filled SalI site of p610ZA to give construct 11 . The fragment for injection was released with PstI and KpnI. The construction of the Hox-2.1 base construct will be described elsewhere; but to insert the end-filled HindIII-NcoI fragment it was digested with KpnI and end-filled, and the two fragments were ligated together to give construct 12. All fragments for injection were purified from agarose gels by using Geneclean or phenol-chloroform and then passed through an Elutip column.

\section{Production and analysis of transgenic mice}

$(\mathrm{CBA} \times \mathrm{C} 57 \mathrm{BL} 10) \mathrm{F}_{1}$ mice were used throughout these experiments as embryo donors, stud males, pseudopregnant females, vasectomized males, and mature females for breeding. Transgenic mice were produced as described by Hogan et al. (1986). Embryos to be stained were fixed in $1 \%$ formaldehyde, $0.2 \%$ glutaraldehyde, $2 \mathrm{mM} \mathrm{MgCl}_{2}, 5 \mathrm{~mm}$ EGTA, and $0.02 \% \mathrm{NP}-40$ in PBS at $4^{\circ} \mathrm{C}$ for $30-90 \mathrm{~min}$ depending on size. They were then washed in three changes of PBS plus $0.02 \% \mathrm{NP}-40$ at room temperature for $30 \mathrm{~min}$ each and stained in the dark in $1 \mathrm{mg} / \mathrm{ml}$ of X-gal, $5 \mathrm{mM} \mathrm{K}_{3} \mathrm{Fe}(\mathrm{CN})_{6}, 5 \mathrm{mM} \mathrm{K}_{4} \mathrm{Fe}(\mathrm{CN})_{6}, 2 \mathrm{mM} \mathrm{MgCl}_{2}, 0.01 \%$ sodium deoxycholate, and $0.02 \% \mathrm{NP}-40$ in PBS at room temperature. This staining protocol sufficed for whole-mount embryos up to $13.5 \mathrm{dpc}$, but after this stage the developing skin acted as a barrier to the fixation and staining. To avoid these problems from 14.5 dpc onward, embryos or tissues were partially dissected to permit full penetration of reagents.

Embryos to be sectioned were fixed further in $4 \%$ paraformaldehyde overnight at $4^{\circ} \mathrm{C}$, dehydrated, and embedded in paraffin wax as described in Wilkinson and Green (1990). Sections were cut $(6 \mu \mathrm{m})$; the sections were dewaxed and counterstained with eosin. In situ analysis was performed as described in Wilkinson and Green (1990). 


\section{Acknowledgments}

During the early stages of this work R.A. was supported by a Royal Society/Schweizerischer Nationalfonds European Science Exchange Programme Fellowship. We thank all of the members of the Krumlauf and Rigby laboratories and Frank Grosveld for helpful discussions, Wendy Hatton for help with histology, and the members of the Biological Services Division for their help with animal husbandry.

The publication costs of this article were defrayed in part by payment of page charges. This article must therefore be hereby marked "advertisement" in accordance with 18 USC section 1734 solely to indicate this fact.

\section{References}

Akam, M. 1987. The molecular basis for metameric pattern in the Drosophila embryo. Development 101: 1-22.

- 1989. Hox and HOM: homologous gene clusters in insects and vertebrates. Cell 57: 347-349.

Bergson, C. and W. McGinnis. 1990. An autoregulatory enhancer element of the Drosophila homeotic gene Deformed. EMBO I. 9: 4287--4297.

Bieberich, C., M. Utset, A. Awgulewitsch, and F. Ruddle. 1990. Evidence for positive and negative regulation of the Hox-3.1 gene. Proc. Natl. Acad. Sci. 87: 8462-8466.

Chisaka, O. and Capecchi, M. R. 1991. Regionally restricted developmental defects resulting from targeted disruption of the mouse homeobox gene hox-1.5. Nature 350: 473-479.

Dollé, P. and D. Duboule. 1989. Two gene members of the murine HOX 5 complex show regional and cell-type specific expression in developing limbs and gonads. EMBO $I$. 8: $1507-1515$.

Dollé, P., J.C. Izpisua-Belmonte, H. Falkenstein, A. Renucci, and D. Duboule. 1989. Coordinate expression of the murine Hox-5 complex homoeobox-containing genes during limb pattern formation. Nature 342: 767-772.

Dony, C. and P. Gruss. 1987. Specific expression of the Hox 1.3 homeo box gene in murine embryonic structures originating from or induced by the mesoderm. EMBO J. 6: 2965-2975.

Dressler, G.R. and P. Gruss. 1989. Anterior boundaries of Hox gene expression in mesoderm-derived structures correlate with the linear gene order along the chromosome. Differentiation 41: 193-201.

Duboule, D. and P. Dollé. 1989. The structural and functional organization of the murine HOX gene family resembles that of Drosophila homeotic genes. EMBO I. 8: 1497-1505.

Gaunt, S.J. 1988. Mouse homeobox gene transcripts occupy different but overlapping domains in embryonic germ layers and organs: A comparison of Hox-3.1 and Hox-1.5. Development 103: 135-144.

Gaunt, S., P. Sharpe, and D. Duboule. 1988. Spatially restricted domains of homeo-gene transcripts in mouse embryos: Relation to a segmented body plan. Development 104: (suppl.) 104: 169-179.

Gaunt, S.J., R. Krumlauf, and D. Duboule. 1989. Mouse homeogenes within a subfamily, Hox-1.4, -2.6 and -5.1 , display similar anteroposterior domains of expression in the embryo, but show stage- and tissue-dependent differences in their regulation. Development 107: 131-141.

Giampaolo, A., D. Acampora, V. Zappavigna, M. Pannese, M. D'Esposito, A. Care, A. Faiella, A. Stornaiuolo, G. Russo, A. Simeone, E. Boncinelli, and C. Peschle. 1989. Differential expression of human HOX-2 genes along the anterior-posterior axis in embryonic central nervous system. Differentiation 40: 191-197.

Graham, A. 1990. "Analysis of murine homeobox genes." Ph.D. thesis, University of London.

Graham, A., P.W. Holland, A. Lumsden, R. Krumlauf, and B.L. Hogan. 1988a. Expression of the homeobox genes Hox 2.1 and 2.6 during mouse development. Curr. Top. Microbiol. Immunol. 137: 87-93.

Graham, A., N. Papalopulu, J. Lorimer, J. McVey, E. Tuddenham, and R. Krumlauf. 1988b. Characterization of a murine homeo box gene, Hox 2.6, related to the Drosophila deformed gene. Genes \& Dev. 2: 1424-1438.

Graham, A., N. Papalopulu, and R. Krumlauf. 1989. The murine and Drosophila homeobox clusters have common features of organisation and expression. Cell 57: 367-378.

Graham, A., M. Maden, and R. Krumlauf. 1991. The murine Hox-2 genes display dynamic dorsoventral patterns of expression during central nervous system development. Development 112: 255-264.

Grosveld, F., G. Blom van Assendelft, D. Greaves, and G. Kollias. 1987. Position-independent, high level expression of the human $\beta$-globin gene in transgenic mice. Cell 51: 975-985.

Hogan, B., F. Costantini, and E. Lacy. 1986. Manipulating the mouse embryo. Cold Spring Harbor Laboratory, Cold Spring Harbor, New York.

Holland, P.W. and B.L. Hogan. 1988. Spatially restricted patterns of expression of the homeobox-containing gene Hox 2.1. during mouse embryogenesis. Development 102: 159174.

Hunt, P., D. Wilkinson, and R. Krumlauf. 1991a. Patterning the vertebrate head: Murine Hox 2 genes mark distinct subpopulations of premigratory and migrating neural crest. Development 112: 43-51.

Hunt, P., I. Whiting, I. Muchamore, H. Marshall, and R. Krumlauf. 1991b. Homeobox genes and models for patterning the hindbrain and branchial arches. Development (suppl. 1) 112: 186-195.

Izpisua-Belmonte, J., H. Falkenstein, P. Dollé, A. Renucci, and D. Duboule. 1991. Murine genes related to the Drosophila $A b d B$ homeotic gene are sequentially expressed during development of the posterior part of the body. EMBO $/$. 10: $2279-2296$.

Kessel, M. and P. Gruss. 1990. Murine developmental control genes. Science 249: 374-379.

- 1991. Homeotic transformations of murine vertebrae and concomitant alteration of Hox codes induced by retinoic acid. Cell 67 (in press).

Kessel, M., R. Balling, and P. Gruss. 1990. Variations of cervical vertebrae after expression of a Hox 1.1 transgene in mice. Cell 61: 301-308.

Kress, C., R. Vogels, W. De Graaff, C. Bonnerot, F. Meijlink, and J. Deschamps. 1990. Hox-2.3 upstream sequences mediate lac $Z$ expression in intermediate mesoderm derivatives of transgenic mice. Development 109: 775-786.

Krumlauf, R., N. Papalopulu, J. Clarke, and N. Holder. 1991. Retinoic acid and altered segmental patterning of the Xenopus hindbrain. Sem. Dev. Biol. 2: 181-188.

Lathe, R., J. Vilotte, and A. Clark. 1987. Plasmid and bacteriophage vectors for excision of intact inserts. Gene 57: 193201.

Lewis, E.B. 1978. A gene complex controlling segmentation in Drosophila. Nature 276: 565-570.

Lufkin, T., A. Dierich, M. LeMeur, M. Mark, and P. Chambon. 1991. Disruption of the Hox-1.6 homeobox gene results in defects in a region corresponding to its rostral domain of 
expression. Cell 66: 1105-1119.

Oliver, G., C.V. Wright, J. Hardwicke, and E.M. De-Robertis. 1988. A gradient of homeodomain protein in developing forelimbs of Xenopus and mouse embryos. Cell 55: 10171024.

Papalopulu, N., P. Hunt, D. Wilkinson, A. Graham, and R. Krumlauf. 1990. Hox-2 homeobox genes and retinoic acid: Potential roles in patterning the vertebrate nervous system. Neurol. Neurobiol. 60: 291-307.

Papalopulu, N., J. Clarke, L. Bradley, D. Wilkinson, R. Krumlauf, and N. Holder. 1991a. Retinoic acid causes abnormal development and segmental patterning of the anterior hindbrain in Xenopus embryos. Development 113 (in press).

Papalopulu, N., R. Lovell-Badge, and R. Krumlauf. 1991b. The expression of the murine Hox-2 genes is dependent on the differentiation pathway and displays collinear sensitivity to retinoic acid in F9 cells and Xenopus embryos. Nucleic Acids Res. 19: 5497-5506.

Puschel, A., R. Balling, and P. Gruss. 1990. Position-specific activity of the Hox 1.1 promoter in transgenic mice. Development 108: 435-442.

- 1991. Separate elements cause lineage restriction and specify boundaries of Hox-1.1 expression. Development 112: 279-287.

Simeone, A., D. Acampora, L. Arcioni, P.W. Andrews, E. Boncinelli, and F. Mavilio. 1990. Sequential activation of HOX2 homeobox genes by retinoic acid in human embryonal carcinoma cells. Nature 346: 763-766.

Simeone, A., D. Acampora, V. Nigro, A. Faiella, M. D'Esposito, A. Stornaiuolo, F. Mavilio, and E. Boncinelli. 1991. Differential regulation by retinoic acid of the homeobox genes of the four HOX loci in human embryonal carcinoma cells. Mech. Dev. 33: 215-227.

Struhl, G. 1984. Splitting the bithorax complex of Drosophila. Nature 308: 454-457.

Stuart, J.J., S.J. Brown, R.W. Beeman, and R.E. Denell. 1991. A deficiency of the homeotic complex of the beetle Tribolium. Nature 350: 72-74.

Tiong, S.Y., J.R. Whittle, and M.C. Gribbin. 1987. Chromosomal continuity in the abdominal region of the bithorax complex of Drosophila is not essential for its contribution to metameric identity. Development 101: 135-142.

Tuggle, C.K., J. Zakany, L. Cianetti, C. Peschle, and M.C. Nguyen-Huu. 1990. Region-specific enhancers near two mammalian homeo box genes define adjacent rostrocaudal domains in the central nervous system. Genes \& Dev. 4: $180-189$.

Wilkinson, D. and J. Green. 1990. In situ hybridization and three-dimensional reconstruction of serial sections. In Postimplantation mouse embryos: A practical approach led. A. Copp and D.L. Cockcroft), pp. 155-171. IRL Press, Oxford.

Wilkinson, D., S. Bhatt, M. Cook, E. Boncinelli, and R. Krumlauf. 1989. Segmental expression of hox 2 homeobox-containing genes in the developing mouse hindbrain. Nature 341: 405-409.

Wright, C.V., K.W. Cho, J. Hardwicke, R.H. Collins, and E.M. De Robertis. 1989. Interference with function of a homeobox gene in Xenopus embryos produces malformations of the anterior spinal cord. Cell 59: 81-93.

Zakany, J., C.K. Tuggle, M.D. Patel, and C.M. Nguyen-Huu. 1988. Spatial regulation of homeobox gene fusions in the embryonic central nervous system. Neuron 1: 679-691. 


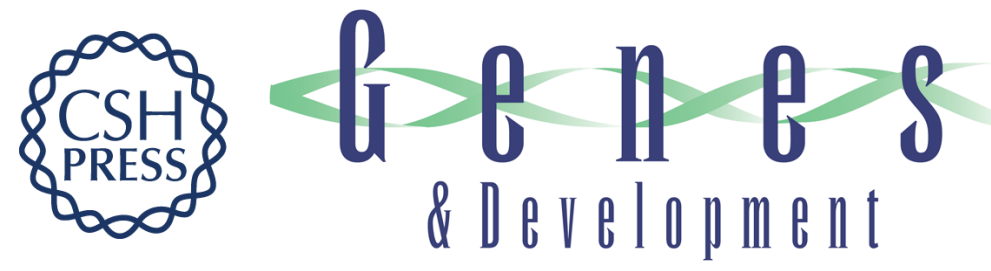

\section{Multiple spatially specific enhancers are required to reconstruct the pattern of Hox-2.6 gene expression.}

J Whiting, H Marshall, M Cook, et al.

Genes Dev. 1991, 5:

Access the most recent version at doi:10.1101/gad.5.11.2048

References This article cites 44 articles, 15 of which can be accessed free at:

http://genesdev.cshlp.org/content/5/11/2048.full.html\#ref-list-1

License

Email Alerting

Service

Receive free email alerts when new articles cite this article - sign up in the box at the top right corner of the article or click here.

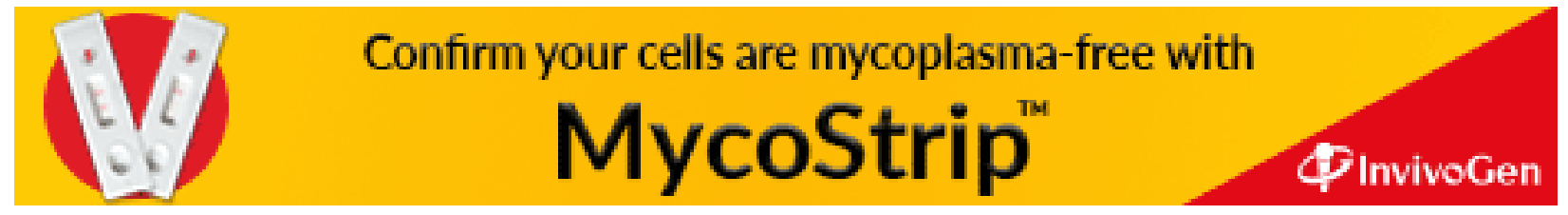

